\title{
EGU2020-18556
}

https://doi.org/10.5194/egusphere-egu2020-18556

EGU General Assembly 2020

(c) Author(s) 2020. This work is distributed under

the Creative Commons Attribution 4.0 License.

\section{A (small) step towards standardisation in rainfall simulation experiments}

\author{
Jorge Isidoro ${ }^{1,7}$, Ian Pattison ${ }^{2}$, Thomas Iserloh ${ }^{3}$, João de Lima ${ }^{4,7}$, Daniel Green ${ }^{5}$, Miriam Marzen ${ }^{3}$, \\ Isabel de Lima ${ }^{4,7}$, Alexandre Silveira ${ }^{6}$, and Ross Stirling ${ }^{5}$ \\ ${ }^{1}$ Civil Engineering Department, Institute of Engineering, University of Algarve, Faro, Portugal \\ ${ }^{2}$ School of Energy, Geoscience, Infrastructure and Society, Heriot-Watt University, Edinburgh, UK \\ ${ }^{3}$ Department of Physical Geography, Trier University, Trier, Germany \\ ${ }^{4}$ Civil Engineering Department, Faculty of Sciences and Technology, University of Coimbra, Coimbra, Portugal \\ ${ }^{5}$ National Green Infrastructure Facility, School of Engineering, Newcastle University, Newcastle upon Tyne, UK \\ ${ }^{6}$ Institute of Science and Technology, Federal University of Alfenas, Poços de Caldas (MG), Brazil \\ ${ }^{7}$ Marine and Environmental Sciences Centre (MARE), Coimbra, Portugal
}

Rainfall simulation is widely used within hydrological and geomorphological sciences and is particularly important in the study of rainfall-runoff, erosion and pollutant transport processes. Rainfall simulators have been applied within laboratory- and field-based studies and have the advantages of enabling controlled and reproducible rainfall event characteristics in relation to rainfall intensity, duration, and drop spectra. The flexibility and advantages of using rainfall simulators to study a wide range of research objectives has resulted in significant diversity in the type, sizing, form, operation and methodologies of rainfall simulators, and an extensive review of rainfall simulator research has led to more than 250 different rainfall simulator setups being identified in the literature. Rainfall simulators come in all different shapes and sizes!

The adaptability of rainfall simulators to study a wide range of research areas of varying scale ultimately results in several issues when comparing results and outputs obtained from different simulator setups. In fact, comparisons between studies can be very difficult, if not impossible, as the different measurement methods, artificial rainfall event characteristics and test conditions result in considerable difficulties when benchmarking results and findings obtained from rainfall simulation experiments. Thus, the scientific community should establish set methodological procedures to allow comparisons between results obtained from different rainfall simulator setups. Harmonization of basic procedures in rainfall simulator based studies in the fields of hydrological and geomorphological sciences would ensure that results between different rainfall simulator studies are comparable, standardised and regulated. The first step in this process involves standardising rainfall simulators design characteristics, whereas further steps should focus on measurement methods and metrics so results can be compared.

This paper aims to bring together current understanding on the use of rainfall simulators within hydrological and geomorphological research, and provide a platform to discuss and enhance 
understanding of the requirements on the standardisation of rainfall simulator based experimental research. This paper also aims to establish an international research community focused on advancing standardisation in rainfall simulation based at different research facilities and institutes, and will kick-start discussions leading up to a future international symposium dealing with these issues (date TBC). Everyone is invited to join this (small) step towards standardisation in rainfall simulation! 